Apidologie, 1971, 2 (3), 221-238.

\title{
LE SPECTRE POLLINIQUE DES MIELS LUXEMBOURGEOIS
}

\author{
Das Pollenbild luxemburgischer Honige
}

\author{
Anna MAURIZIO
}

Liebefeld-Bern (Suisse)

\section{SUMMARY}

\section{THE POLLEN SPECTRUM OF HONEYS FROM LUXEMBURG}

In the present investigation 130 Luxemburg honeys from the years 1945, 1947, 1954. 1966 and 1967 was examined by pollen analysis. The Tables and Figures show the results : the distribution of the samples in the 12 cantons of Luxemburg (Table 1, Fig. 1); the numbers of different pollen types found in each honey (Table 3, Fig. 2); the detailed pollen spectrum of all samples (Table 2) ; the frequency of occurence of the pollen types in the honeys (Table 4, Fig. 3); and the distribution of the 17 predominant pollen types and of honeydew elements in the samples from different cantons and years (Table $5 a, 5 b, 6 a, 6 b)$. The characteristic pollen combinations in Luxemburg honeys are discussed, and also differences in the pollen spectra of honeys from earlier and later years.

\section{RESUMÉ}

La présente communication analyse les résultats de l'examen microscopique de 130 échantillons de miels luxembourgeois des années $1945,1947,1954,1966$ et 1967 . Les résultats sont présentés dans des tableaux et des planches. Ils montrent la répartition de la prise d'échantillons dans les 12 cantons du Luxembourg (tableau 1, fig. 1), le nombre des différentes formes de pollen trouvées par miel (tableau 3, fig. 2), le spectre pollinique détaillé de tous les miels analysés (tableau 2), la fréquence de la présence des formes de pollens dans le spectre (tableau 4 , fig. 3), ainsi que la répartition des 17 formes de pollens prédominantes et des indicateurs de miellat dans les échantillons des divers cantons et d'années différentes (tableaux 5a, b, 6a, b). Les combinaisons de pollen caractéristiques pour les miels luxembourgeois sont commentées à la lumière de ces résultats, ainsi que les différences entre les miels des récoltes anciennes et récentes.

\section{INTRODUCTION}

L'analyse microscopique des miels luxembourgeois promet d'être pleine d'intérêt quand on considère la situation géographique, le climat, l'agriculture 
et l'économie forestière du pays. Cela d'autant plus que l'excellente organisation du contrôle des miels au Luxembourg offre une garantie sûre pour des prises d'échantillons irréprochables et des indications exactes sur la provenance des miels (Luxembourg, Résolution sur le miel, 1966).

L'analyse devait contribuer à éclaircir les questions suivantes :

- De quelles plantes mellifères proviennent essentiellement les miels luxembourgeois, c'est-à-dire quelles sont les formes polliniques prédominantes, et de quelles formes sont-elles accompagnées?

- Dans quelles proportions le miellat entre-t-il dans les miels luxembourgeois?

- Quels types caractéristiques de miels trouve-t-on au Luxembourg et comment sont-ils répartis dans les différentes régions?

- Existe-t-il des différences dans le spectre pollinique entre les miels de récoltes anciennes et récentes qui peuvent indiquer une évolution des conditions de récolte?

\section{ECHANTILLONS DE MIEL, MÉTHODE D'ANALYSE ET DESCRIPTION DES RESULTATS}

130 échantillons de miel au total ont été examinés, provenant de ruchers situés dans 72 localités, réparties dans les 12 cantons du Luxembourg. A peu près la moitié des échantillons concerne les années 1966 et 1967 (69 échantillons $=53 \%$ ) les autres se répartissent sur les années 1945, 1947 et 1954 (61 échantillons $=47 \%$, voir tableau 1 et fig. 1). Toutes les régions du pays ont été prises en considération dans l'analyse, les échantillons provenant de tous les cantons.

TABleaU 1. - Nombre et pourcentage des miels luxembourgeois examinés (classés par cantons et années de récolte)

TaBelle 1. - Gesamtzahl und prozentualer Anteil der untersuchten luxemburgischen Honige, angeordnet nach Kantonen und Erntejahren

\begin{tabular}{|c|c|c|c|c|c|c|c|}
\hline \multirow{2}{*}{\multicolumn{2}{|c|}{$\begin{array}{l}\text { Canton } \\
\text { Kanton }\end{array}$}} & \multirow{3}{*}{$\begin{array}{c}1945 \\
(1947)\end{array}$} & \multicolumn{3}{|c|}{$\begin{array}{c}\text { Année de récolte } \\
\text { Erntejahr }\end{array}$} & \multicolumn{2}{|c|}{ Total } \\
\hline & & & 1954 & 1966 & 1967 & \multirow{2}{*}{$\begin{array}{c}\begin{array}{c}\text { Nombre } \\
\text { Anzahl }\end{array} \\
16\end{array}$} & \multirow{2}{*}{$\frac{\%}{12,3}$} \\
\hline 1 & Luxembourg.......... & & 4 & 4 & 7 & & \\
\hline 2 & Mersch . . . . . . . . . . & - & 3 & 2 & 4 & 9 & 6,9 \\
\hline 3 & Capellen ............ & - & 6 & 1 & 2 & 9 & 6,9 \\
\hline 4 & $\operatorname{Esch} \ldots \ldots \ldots \ldots \ldots$ & 2 & 5 & 2 & 4 & 13 & 10,0 \\
\hline 5 & Remsch $\ldots \ldots \ldots \ldots$ & 1 & 7 & 2 & 2 & 12 & 9,2 \\
\hline 6 & Grevenmacher $\ldots \ldots \ldots$ & $\mathbf{l}$ & 3 & 2 & 1 & 7 & 5,4 \\
\hline 7 & Echternach $\ldots \ldots \ldots$ & 1 & 6 & 2 & 5 & 14 & 10,8 \\
\hline 8 & Diekirch . . . . . . . . & - & 1 & 2 & 5 & 8 & 6,2 \\
\hline 9 & Vianden $\ldots \ldots \ldots \ldots$ & - & 1 & 1 & - & 2 & 1,5 \\
\hline 10 & Redingen $\ldots \ldots \ldots \ldots$ & 3 & 1 & 4 & 12 & 20 & 15,4 \\
\hline 11 & Wiltz $\ldots \ldots \ldots \ldots \ldots$ & 3 & 8 & 1 & - & 12 & 9,2 \\
\hline \multirow[t]{3}{*}{12} & Clerf............... & 2 & 2 & 1 & 3 & 8 & 6,2 \\
\hline & TotaL $\ldots \ldots \ldots \ldots \ldots$ & 14 & 47 & 24 & 45 & 130 & \\
\hline & $\% \ldots \ldots \ldots \ldots \ldots$ & 10,7 & 36,2 & 18,5 & 34,6 & & \\
\hline
\end{tabular}




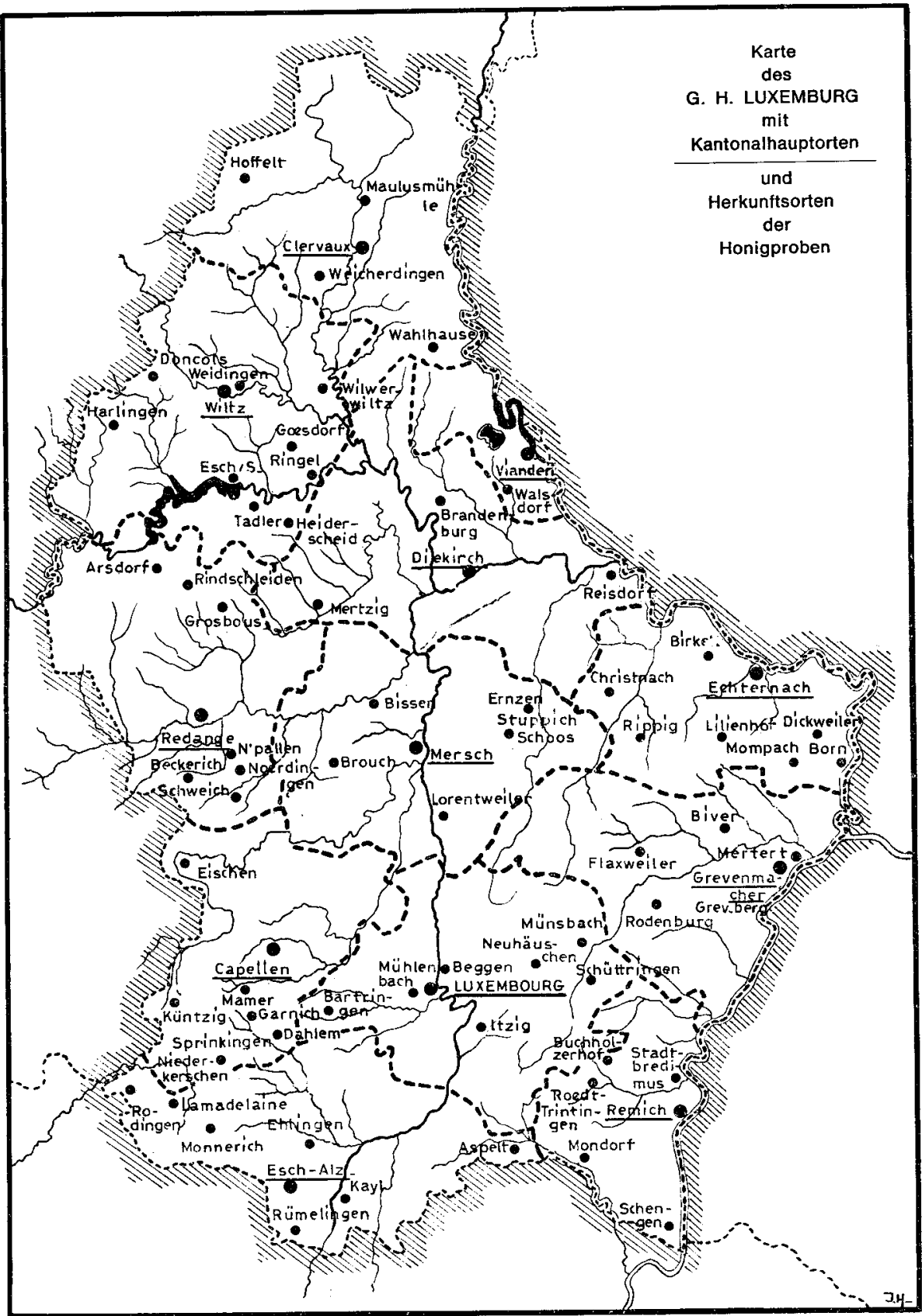

Fig. 1. - Répartition des échantillons de miel étudiés dans les 12 cantons du G. D. de Luxembourg. Aвв. 1. - Verteilung der untersuchten Honige in den 12 Kantonen des G.-H. Luxemburg. 
Les miels ont été préparés d'après la méthode décrite par la Commission internationale de botanique apicole de l'U.I.S.B., (Commission internationale de botanique apicole 1962, 1970).

Pour chaque miel, 100 à 200 grains de pollen ainsi que les indicateurs de miellat qui les accompagnaient, ont été déterminés et comptés. Les résultats du dénombrement ont été ensuite classés en groupes, d'après leur fréquence. Pour le pollen, 3 groupes sont admis : le pollen dominant (plus de $45 \%$ du pollen total), le pollen d'accompagnement (16-45 \%) et le pollen isolé $(1-15 \%)$; les indicateurs de miellat sont répartis en 5 groupes, d'après la relation entre les indicateurs de miellat (IM) et les grains de pollen (P) : pas de miellat (IM /P 0-0, 1), peu $(0,11-0,5)$, quantité moyenne $(0,51-1,0)$ grande quantité $(1,01-2,0)$, très grande quantité (plus de 2,0).

Les résultats de l'analyse sont reportés dans les tableaux et figures qui suivent.

\section{RESULTATS DE L'ANALYSE}

\section{I. - Spectre pollinique des miels luxembourgeois}

\section{1. - Richesse en formes des miels luxembourgeois}

Un regard sur le tableau 2 montre que le spectre pollinique des miels luxembourgeois est relativement riche en formes diverses. Dans le tableau 3, fig. 2, les miels sont placés d'après le nombre de formes de pollen qui s'y trouvent.

Plus de $60 \%$ des miels examinés appartiennent à la classe des 11-15 formes de pollen, $31 \%$ à celle des 6-10 formes, c'est-à-dire que $95 \%$ à peu près de tous les miels contiennent 6-15 formes différentes de pollen. La moyenne est de 12,7 formes de pollen par miel. Les miels très riches en formes de pollen (16-20) et très pauvres (1-5) sont rares (en tout $5 \%$ ). A ce point de vue, il n'y a pas de différence sensible entre les cantons. Les miels luxembourgeois restent ainsi dans le cadre de la plupart des miels d'Europe centrale qui contiennent aussi en moyenne 10-15 formes de pollen, ce qui indique un choix de sources de récolte plus riche en espèces qu'en nectar. Les miels très riches en formes de pollen, 20 à 30, parfois plus de 40, se trouvent surtout dans le sud de l'Europe et dans les régions de montagne; ceux très pauvres en formes de pollen (1-5) dans les régions à récolte monoflorale sur un petit nombre d'espèces sauvages ou cultivées très mellifères, ou avec récolte unilatérale de miellat.

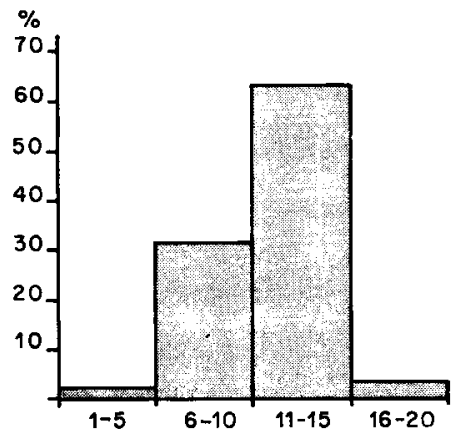

Fic. 2. - Nombre des différentes formes de pollen dans les miels luxembourgeois. Horizontalement: nombre des formes par miel.

Aв8. 2. - Anzahl verschiedener Pollenformen in den luxemburgischen Honigen. Abszisse : Anzahl Formen je Honig. 


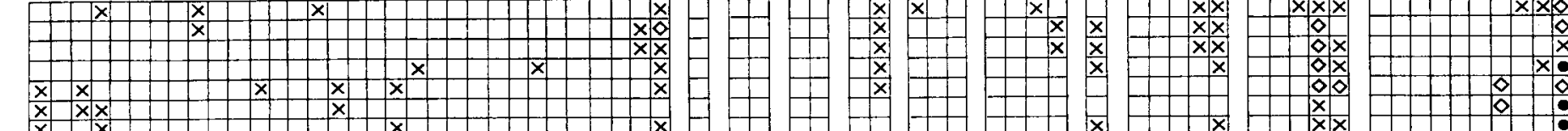

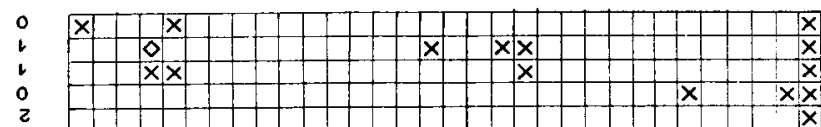

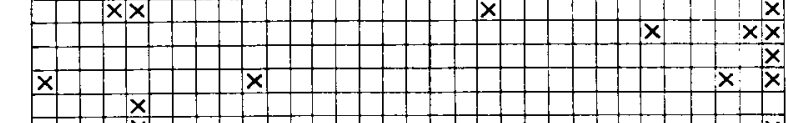

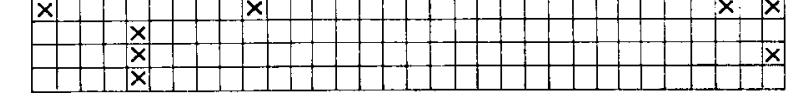

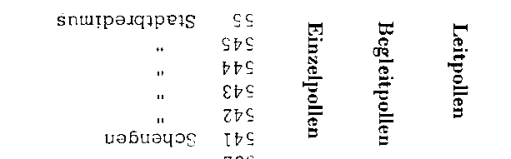


$7^{2}$ 
TABLEAU 3. - Nombre des différentes formes

de pollen dans les miels luxembourgeois

Tabelle 3. - Anzahl verschiedener Pollenformen in den luxemburgischen Honigen

\begin{tabular}{|c|c|c|c|c|}
\hline \multirow{2}{*}{$\begin{array}{l}\text { Canton } \\
\text { Kanton }\end{array}$} & \multicolumn{4}{|c|}{$\begin{array}{l}\text { Nombre de formes de pollen par miel } \\
\text { Anzahl der Pollenformen je Honig }\end{array}$} \\
\hline & $1-5$ & $6-10$ & 11-15 & $16-20$ \\
\hline 1 & - & 4 & 12 & - \\
\hline 2 & - & 3 & 5 & 1 \\
\hline 3 & - & 3 & 6 & - \\
\hline 4 & - & 5 & 8 & - \\
\hline 5 & - & 3 & 8 & 1 \\
\hline 6 & - & I & 6 & - \\
\hline 7 & - & 5 & 9 & - \\
\hline 8 & - & 1 & 6 & 1 \\
\hline 9 & - & - & 1 & 1 \\
\hline 10 & 2 & 10 & 8 & - \\
\hline 11 & - & 2 & 9 & 1 \\
\hline 12 & - & 4 & 4 & - \\
\hline Total & 2 & 41 & 82 & 5 \\
\hline$\%$ & 1,5 & 31,5 & 63,2 & 3,8 \\
\hline
\end{tabular}

\section{2. - Les formes de pollen dans le spectre des miels luxembourgeois}

Dans les 130 miels examinés, 67 formes de pollen ont pû être déterminées, en partie jusqu'à l'espèce, en partie seulement jusqu'à la forme, au groupe ou à la famille. On entend par “ groupe » par exemple “ trèfle blanc », un cercle de formes qui comprend, à côté de Trifolium repens, Trifolium hybridum et éventuellement $d$ 'autres espèces de trèfles à pollen semblable; on entend par “ forme » des formes de pollen d'un certain type, qui appartiennent à différentes espèces ou genres de la même famille, par exemple certaines labiées, composées, etc. Le tableau 4 et la figure 3 montrent la diffusion des pollens dans les miels. Le tableau indique la fréquence des espèces de pollen, ordonnées d'après les familles de plantes ; sur la planche les formes sont notées d'après le pourcentage ; les fleurs nectarifères, les fleurs à pollen sans nectar et les anémophiles sont signalées spécialement. Ces tableaux montrent que le groupe trèfle blanc joue un rôle prédominant dans les miels luxembourgeois. Ce groupe se trouve dans 127 des 130 échantillons $(97,7 \%)$ c'est-à-dire qu'il fait partie pratiquement de tous les miels luxembourgeois. A côté du trèfle blanc, il y a deux représentants de la même famille qui sont fréquents, le trèfle rouge (Trifolium pratense, 
Tableau 4, - Répartition des différentes espèces de pollen dans les miels luxembourgeois (nombre et pourcentage, classés d'après les familles de plantes)

Tabelce 4. - Verbreitung der verschiedenen Pollenformen in den luxemburgischen Honigen (Zahl und prozentualer Anteil, nach Pfanzenfamilien geordnet)

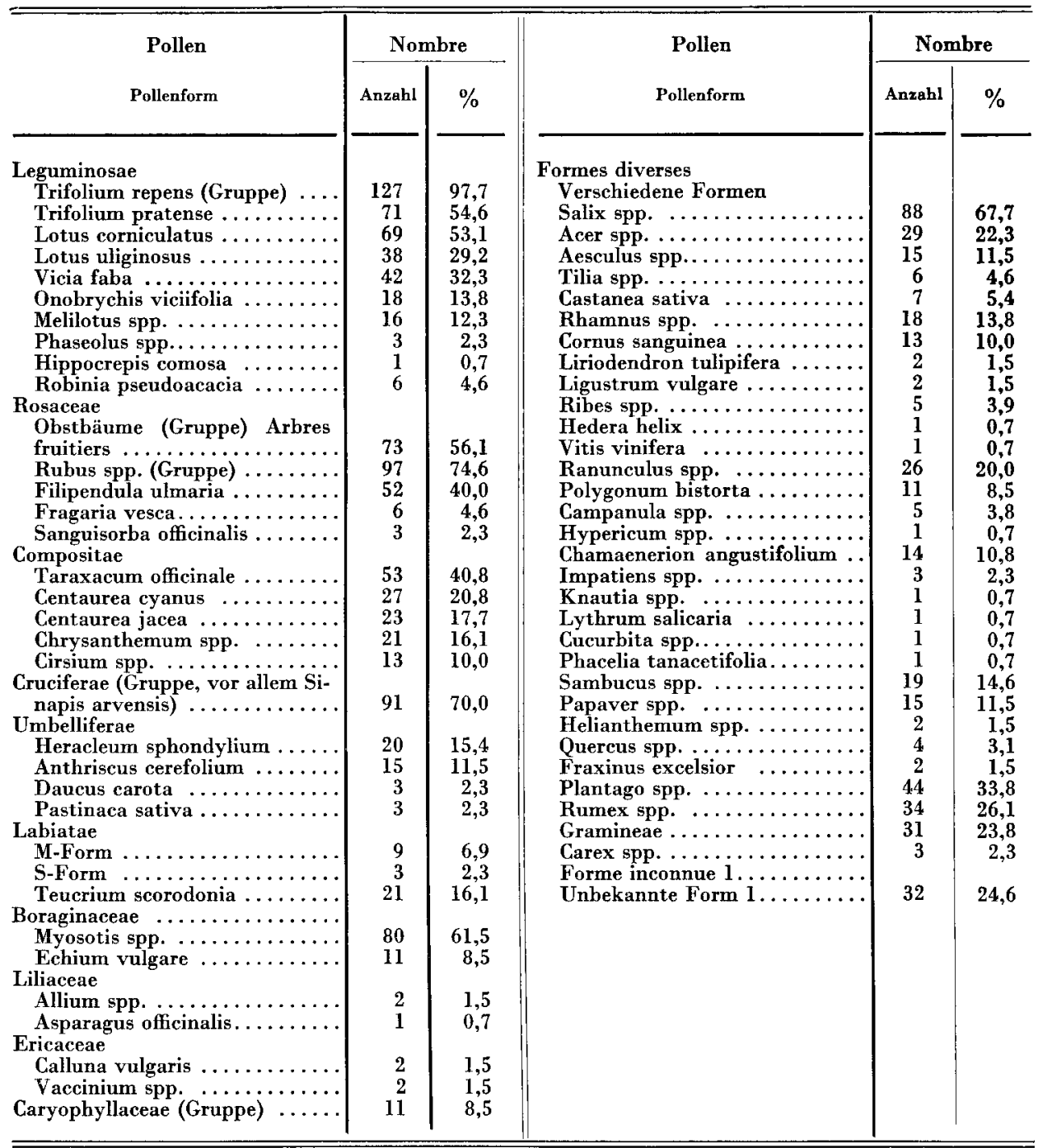

$54 \%$ ) et le lotier (Lotus corniculatus, $53 \%$ ). Dans les formes de pollen fréquentes, qu'on retrouve dans plus de la moitié des miels luxembourgeois, on peut compter les framboisiers (groupe Rubus, comptant de nombreuses espèces, dont les framboisiers et les ronces) les crucifères (surtout Sinapis arvensis) Salix, Myosotis (2 espèces différentes avec pollen de grosseur différente) et les arbres fruitiers (fruits à pépins et à noyaux, espèces de Pyrus et Prunus). Toutes ces plantes sont mellifères et entomophiles, et comptent, à part Myoso- 


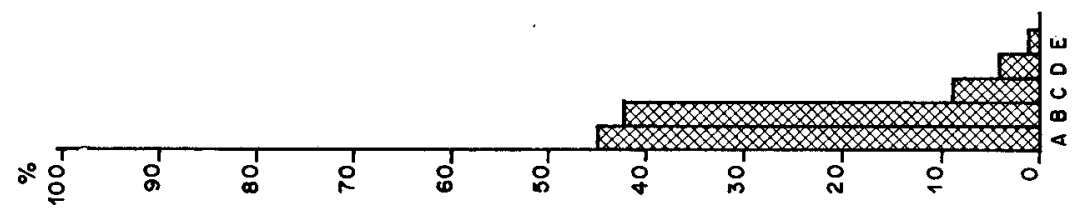

1. Trifolium repens

2. Rubus

3. Cruciferae

4. Salix

5. Myosotis

6. Arbres fruitiers

Obstbäume

7. Trifolium pratense

8. Lotus corniculatus

9. Taraxacum officinale

10. Filipendula ulmaria

11. Plantago

12. Vicia faba

13. Lotus uliginosus

14. Rumex

15. Forme inconnue 1 Unbekannte Form 1

16. Gramineae

17. Acer

18. Centaurea cyanus

19. Ranunculus

20. Centaurea jacea

21. Chrysanthemum leuc.

22. Teucrium scorodonia

23. Heracleum sphondylium

24. Sambucus

25. Onobrychis viciifolia

26. Rhamnus

27. Melilotus

28. Aesculus hippocastanum

29. Papaver

30. Anthriscus cerefolium

31. Chamaenerion angustif.

32. Cornus sanguinea

33. Cirsium, Carduus

34. Caryophyllaceae

35. Echium

36. Polygonum bistorta
37. Labiatae M.

38. Castanea sativa

39. Robinia pseudoacacia

40. Tilia

41. Fragaria vesca

42. Campanula

43. Ribes

44. Quercus

45. Phaseolus

46. Sanguisorba officinalis

47. Carex

48. Pastinaca sativa

49. Daucus carota

50. Labiatae S.

51. Impatiens

52. Hippocrepis comosa

53. Helianthemum

54. Calluna vulgaris

55. Vaccinium

56. Ligustrum

57. Fraxinus

58. Liriodendron tulipifera

59. Asparagus officinalis

60. Allium

61. Vitis vinifera

62. Phacelia tanacetifolia

63. Hedera helix

64. Hypericum

65. Lythrum salicaria

66. Knautia

67. Cucurbita

A. Pas de miellat. Kein Hgt.

B. Peu de miellat. Wenig Hgt.

C. Quant. moyenne. Z. viel.

D. Gde. quantité. Viel

E. Très gde. quantité. S. viel.

Plantes entomophiles dépourvues de nectaires Insektenblütige, nektarlose Pflanzen

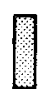

Plantes anémophiles Windblitige Pflanzen

Plantes nectarifères Nektarpflanzen
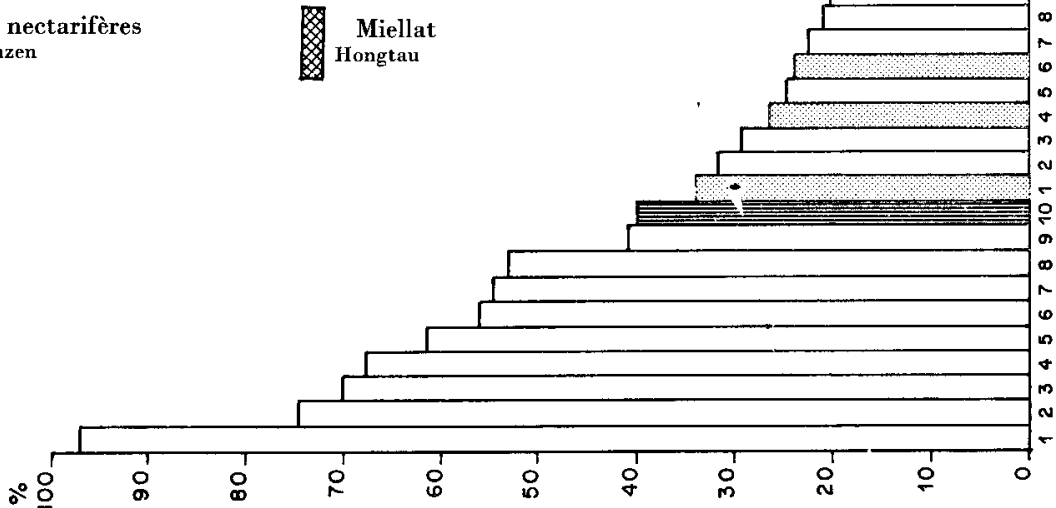

FIg. 3. - Rêpartition des diffërentes espèces de pollen et (à droite) des indicateurs de miellat dans les miels luxembourgeois (en \% des échantillons étudiés).

Aвв. 3. - Verbreitung der verschiedenen Pollenformen und der Honigtaubestandteile in den luxemburgischen Honigen (in \% der untersuchten Proben). 
tis, dans toute l'Europe centrale, parmi les plus importantes sources de nectar.

Les espèces qui se trouvent dans 20-40\% des miels examinés forment un deuxième groupe. Il y a là plusieurs fleurs mellifères connues comme Taraxacum officinale, Vicia faba, Lotus uliginosus, Acer, Teucrium scorodonia et Centaurea cyanus; mais à part ça aussi des anémophiles comme les graminées, Rumex et Plantago ainsi que Filipendula ulmaria, une fleur à pollen qui ne produit point ou très peu de nectar. Dans ce groupe se trouve aussi une forme de pollen qui n'a pas pû être déterminée jusqu'à maintenant et qui est classée sous « forme inconnue 1 m. Dans un autre groupe se trouvent 14 formes de pollen qui sont représentées dans 10-20\% des miels, parmi lesquelles de bonnes fleurs mellifères comme Heracleum sphondylium, Anthriscus cerefolium, Melilotus, Onobrychis, Aesculus, Centaurea jacea, Chrysanthemum leucanthemum, Cirsium, Cornus sanguinea et Rhamnus frangula.

Parmi les fleurs à pollen entomophiles qui n'ont pas de nectar, on trouve fréquemment, à côté de Filipendula ulmaria, des espèces de Papaver et Sambucus ; le pollen de Ranunculus est remarquablement répandu. Les espèces de Ranunculus possèdent toutefois des nectaires, mais ne sont en général pas mellifères, alors qu'elles donnent beaucoup de pollen.

Toutes les autres formes, parmi lesquelles des sources de nectar reconnues comme Tilia, Robinia, Castanea sativa, Calluna vulgaris et des labiées de la forme Salvia et Thymus, restent cantonnées dans moins du $10 \%$ des échantillons. Il est intéressant de constater qu'on trouve accidentellement dans les miels luxembourgeois des formes de pollen plutôt rares dans les miels d'Europe centrale, comme Impatiens, Asparagus, Vitis, Cucurbita, Phacelia, Lythrum, Hedera et Liriodendron. Les grains de pollen d'arbres et d'arbustes comme Quercus, Fraximus, Ligustrum, Ribes et les formes déjà citées, Salix, Rhamnus, Acer, Aesculus, Cornus sanguinea et Sambucus sont assez fréquents.

\section{3. - Formes de pollen prédominantes dans les miels luxembourgeois}

La majorité des formes de pollen des miels luxembourgeois se situe au niveau des pollens isolés, une petite partie seulement représente les pollens dominants et d'accompagnement. Les 17 formes de pollen qui prédominent quantitativement sont groupées dans les tableaux 5 a et $b$, et classées par cantons et années de récolte. Le tableau 5 a montre que la proportion des formes ne correspond pas toujours à leur répartition.

Le groupe trèfle blanc joue un rôle prédominant, étant représenté presque partout et en nombre. Le trèfle blanc trouvé dans 127 miels était dans 54 cas (42\%) pollen dominant et dans 50 cas (38\%) pollen d'accompagnement, c'est-à-dire que cette forme est représentée dans le $80 \%$ des échantillons à peu près, en quantités de plus de $15 \%$. Comme le trèfle blanc appartient aux 


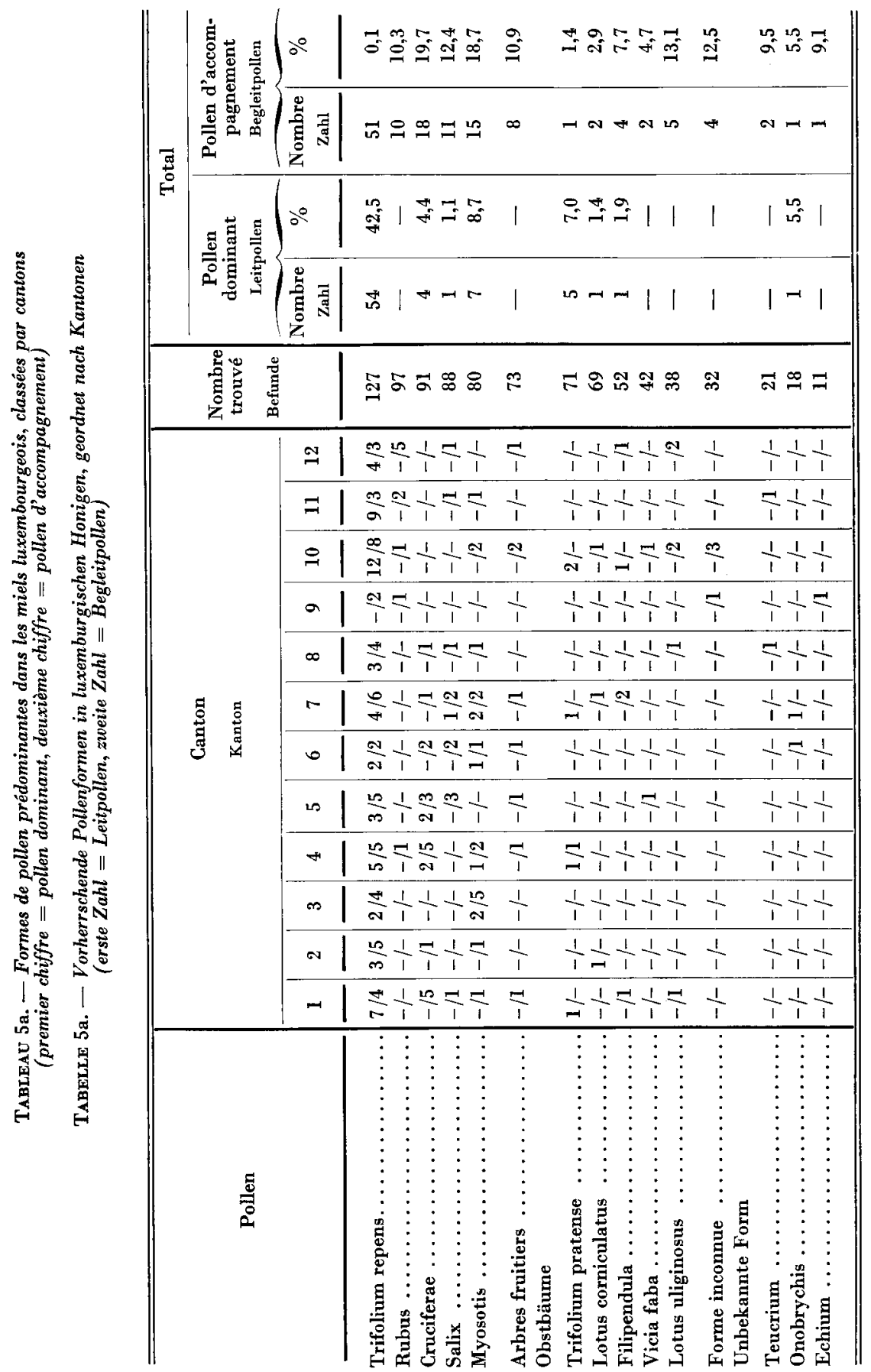


Tableau 5 b. - Formes de pollen prédominantes dans les miels luxembourgeois, classées d'après les années de récolte

(1947 avec 1945, premier chiffre $=$ pollen dominant, deuxième chiffre = pollen d'accompagnement)

Tabelle 5 b. - Vorherrschende Pollenformen in luxemburgischen Honigen, geordnet nach Erntejahren

(1947 bei 1945, erste Zahl = Leitpollen, zweite Zahl $=$ Begleitpollen $)$

\begin{tabular}{|c|c|c|c|c|c|c|c|c|c|}
\hline \multirow[t]{3}{*}{ Pollen } & \multicolumn{4}{|c|}{$\begin{array}{c}\text { Année de récolte } \\
\text { Erntejahr }\end{array}$} & \multicolumn{5}{|c|}{ Total } \\
\hline & \multirow[b]{2}{*}{1945} & \multirow[b]{2}{*}{1954} & \multirow[b]{2}{*}{1966} & \multirow[b]{2}{*}{1967} & \multirow{2}{*}{$\begin{array}{l}\text { Nombre } \\
\text { trouvé } \\
\text { Befunde }\end{array}$} & \multicolumn{2}{|c|}{$1945 / 1954$} & \multicolumn{2}{|c|}{$1966 / 1967$} \\
\hline & & & & & & $\begin{array}{c}\text { Nombre } \\
\text { Zahl }\end{array}$ & $\%$ & $\begin{array}{c}\text { Nombre } \\
\text { Zahl }\end{array}$ & $\%$ \\
\hline Trifolium repens $\ldots .$. & $6 / 6$ & $8 / 19$ & $7 / 16$ & $33 / 10$ & 127 & $14 / 25$ & $11,0 / 19,6$ & $40 / 26$ & $31,5 / 20,5$ \\
\hline Rubus ............ & $-/ 2$ & $-/ 3$ & -14 & $-/ 1$ & 97 & $-/ 5$ & $-/ 5,2$ & $-/ 5$ & $-/ 5,2$ \\
\hline Cruciferae ... & $-1-$ & $3 / 12$ & $-/ 1$ & $1 / 5$ & 91 & $3 / 12$ & $3,9 / 13,2$ & $1 / 6$ & $1,1 / 6,5$ \\
\hline Salix ..... & $-/ 3$ & $1 / 5$ & $-/ 3$ & $-1-$ & 88 & $1 / 8$ & $1,1 / 9,1$ & $-/ 3$ & $-/ 3,4$ \\
\hline Myosotis ......... & $-/ 2$ & $6 / 7$ & $1 / 4$ & $-/ 2$ & 80 & $6 / 9$ & $7,5 / 11,2$ & $1 / 6$ & $1,2 / 7,5$ \\
\hline $\begin{array}{l}\text { Arbres fruitiers } \ldots \cdots \cdots \\
\text { Obstbäume }\end{array}$ & $-/ 1$ & $-1-$ & $-/ 1$ & -16 & 73 & $-/ 1$ & $-/ 1,4$ & $-/ 7$ & $-/ 9,6$ \\
\hline Trifolium pratense....... & $4 / 1$ & $1 /-$ & $-1-$ & $-1-$ & 71 & $5 / 1$ & $7,0 / 1,4$ & $-1-$ & $-1-$ \\
\hline Lotus corniculatus........ & $-1-$ & $-/ 1$ & $-1-$ & $1 / 1$ & 69 & $-/ 1$ & $-/ 1,4$ & $1 / 1$ & $1,4 / 1,4$ \\
\hline Filipendula $\ldots \ldots \ldots \ldots$. & $-1-$ & $1 / 1$ & $-/ 2$ & $-/ 1$ & 52 & $1 / 1$ & $1,9 / 1,9$ & $-/ 3$ & $-/ 5,7$ \\
\hline Vicia faba $\ldots \ldots \ldots \ldots$ & $-1-$ & $-1-$ & $-/ 1$ & $-/ 1$ & 42 & $-1-$ & $-1-$ & $-/ 2$ & $-/ 4,7$ \\
\hline Lotus uliginosus......... & $-1-$ & $-/ 1$ & $-1-$ & $-/ 4$ & 38 & $-/ 1$ & $-/ 2,6$ & -14 & $-/ 10,5$ \\
\hline $\begin{array}{l}\text { Forme inconnue } 1 \ldots \ldots \\
\text { Unbekannte Form } 1\end{array}$ & $-1-$ & $-1-$ & $-/ \mathbf{3}$ & $-/ 1$ & 32 & $-1-$ & $-1-$ & $-/ 4$ & $-/ 12,5$ \\
\hline Teucrium .......... & $-1-$ & $-/ 1$ & $-1-$ & $-/ 1$ & 21 & $-/ 1$ & $-14,7$ & $-/ 1$ & $-/ 4,7$ \\
\hline Onobrychis $\ldots \ldots \ldots \ldots$. & $1 /-$ & $-/ 1$ & $-1-$ & $-1-$ & 18 & $1 / 1$ & $5,5 / 5,5$ & $-1-$ & $-1-$ \\
\hline Echium $\ldots \ldots \ldots \ldots$ & $-1-$ & $-/ 1$ & $-1-$ & $-1-$ & 11 & $-/ \mathbf{1}$ & $-/ 9,0$ & $-1-$ & $-1-$ \\
\hline
\end{tabular}

formes « normalement » représentées dans le miel, on peut dire qu'il compte au Luxembourg pour une des plus importantes sources de nectar.

Les proportions sont différentes avec les autres légumineuses, les crucifères, les rosacées, les espèces de Salix, Teucrium et les anémophiles. Le trèfle rouge par exemple se trouve dans plus de la moitié des miels luxembourgeois, il n'était toutefois pollen dominant que dans 5 cas, et pollen d'accompagnement dans un seul cas. Les quatre échantillons qu'on peut considérer comme miels purs de trèfle rouge sont de la récolte 1945 et étaient désignés comme tels par l'expéditeur. Le cas est semblable pour les deux espèces de Lotus, Onobrychis et Vicia faba, les espèces de Rubus, les arbres fruitiers et les labiées, qu'on trouve très souvent dans le spectre pollinique, mais qui forment très rarement des miels monofloraux. Les crucifères et les Salix pourraient jouer un rôle plus important, étant représentés dans $20 \%$, resp. $12 \%$ à peu près des échantillons comme pollen d'accompagnement, se haussant parfois jusqu'au pollen dominant. Les nombreux pollens dominants et d'accompagnement de Myosotis doivent être mis à part, car les espèces de Myosotis appartiennent aux formes 
sur-représentées dans le miel, et ces résultats demandent une correction. Les grains de Filipendula doivent aussi être interprétés avec prudence. On sait encore peu de choses sur la manière dont le pollen de Papaver et Sambucus pénètrent dans le miel; les pollens anémophiles sont souvent liés au miellat.

\section{II. - Indicateurs de miellat dans les miels luxembourgeois}

Une partie des miels analysés contient des indicateurs de miellat plus ou moins nombreux. Ceux-ci sont notés dans le tableau 2 pour les échantillons détaillés; ils figurent, classés par cantons, sur le tableau 6a, par années de récolte sur le tableau $6 \mathrm{~b}$ et sur la fig. 3 (partie droite) pour la fréquence. D'après ces tableaux, 59 échantillons (45\%) contiennent si peu de spores de champignons qu'ils peuvent être classés comme purs miels de fleurs. 54 échantillons (41 \%) contiennent « peu » d'indicateurs de miellat et peuvent être définis comme miels de fleurs à faible proportion de miellat. Il n'y a que 17 échantillons (13\%) qui contiennent de plus grandes quantités d'indicateurs

Tableau 6. - Part du miellat dans les miels luxembourgeois, classées. $a$ - d'après les cantons, $b$ - d'après les années de récolte

TaBelle 6. - Honigtauanteil in luxemburgischen Honigen $a$ - nach Kantonen, $b$ - nach Erntejahren geordnet

6 a

\begin{tabular}{|c|c|c|c|c|c|c|c|c|c|}
\hline \multirow{2}{*}{$\begin{array}{l}\text { Canton } \\
\text { Kanton }\end{array}$} & \multicolumn{9}{|c|}{$\begin{array}{c}\text { Miellat } \\
\text { Honigtauanteil }\end{array}$} \\
\hline & $\begin{array}{c}\text { pas de } \\
\text { miellat } \\
\text { kein }\end{array}$ & $\begin{array}{l}\text { peu } \\
\text { wenig }\end{array}$ & $\begin{array}{l}\text { Total } \\
\text { Sunme }\end{array}$ & $\%$ & $\begin{array}{c}\text { quantité } \\
\text { moyenne } \\
\text { ziemlich viel }\end{array}$ & $\begin{array}{c}\text { grande } \\
\text { quantité } \\
\text { viel }\end{array}$ & $\begin{array}{l}\text { très grande } \\
\text { quantité } \\
\text { sehr viel }\end{array}$ & Total & $\%$ \\
\hline 1 & 11 & 3 & 14 & 87,5 & - & 1 & l & 2 & 12,5 \\
\hline 2 & 1 & 5 & 6 & 66,6 & 2 & 1 & - & 3 & 33,4 \\
\hline 3 & 4 & 4 & 8 & 88,8 & 1 & - & - & 1 & 11,2 \\
\hline 4 & 8 & 4 & 12 & 86,1 & - & 1 & - & 1 & 13,9 \\
\hline 5 & 7 & 4 & 11 & 91,6 & 1 & - & - & 1 & 8,4 \\
\hline 6 & 2 & 5 & 7 & 100 & - & - & - & - & - \\
\hline 7 & 6 & 6 & 12 & 85,7 & 2 & - & - & 2 & 14,3 \\
\hline 8 & 2 & 4 & 6 & 75,0 & 2 & - & - & 2 & 25,0 \\
\hline 9 & 2 & - & 2 & 100 & - & - & - & - & - \\
\hline 10 & 11 & 7 & 18 & 90,0 & 1 & 1 & - & 2 & 10,0 \\
\hline 11 & 4 & 7 & 11 & 91,6 & 1 & - & - & 1 & 8,4 \\
\hline 12 & 1 & 5 & 6 & 75,0 & 2 & - & - & 2 & 25,0 \\
\hline TotaL & 59 & 54 & 113 & 86,9 & 12 & 4 & 1 & 17 & 13,1 \\
\hline$\%$ & 45,4 & 41,5 & & & 9,2 & 3,1 & 0,7 & & \\
\hline
\end{tabular}


de miellat, dont 4 « en grande quantité » et 1 « en très grande quantité ». Plus de $85 \%$ des miels analysés ne contient pas ou contient très peu de miellat, et les véritables miels de forêt sont très rares.

$6 \mathrm{~b}$

\begin{tabular}{|c|c|c|c|c|c|c|c|c|c|}
\hline \multirow{2}{*}{$\begin{array}{l}\text { Année } \\
\text { de } \\
\text { récolte } \\
\text { Erntejabr }\end{array}$} & \multicolumn{9}{|c|}{$\begin{array}{c}\text { Miellat } \\
\text { Honigtauanteil }\end{array}$} \\
\hline & $\begin{array}{c}\text { pas de } \\
\text { miellat } \\
\text { kein }\end{array}$ & $\begin{array}{l}\text { peu } \\
\text { wenig }\end{array}$ & $\begin{array}{l}\text { Total } \\
\text { Summe }\end{array}$ & $\%$ & $\begin{array}{c}\text { quantité } \\
\text { moyenne } \\
\text { ziemlich viel }\end{array}$ & $\begin{array}{c}\text { grande } \\
\text { quantité } \\
\text { viel }\end{array}$ & $\begin{array}{l}\text { très grande } \\
\text { quantité } \\
\text { sehr viel }\end{array}$ & Total & $\%$ \\
\hline 1945 & 4 & 7 & 11 & 78,5 & 2 & 1 & - & 3 & 21,5 \\
\hline 1954 & 19 & 22 & 41 & 87,2 & 3 & 2 & 1 & 6 & 12,8 \\
\hline 1966 & 12 & 7 & 19 & 79,1 & 4 & 1 & - & 5 & 20,9 \\
\hline 1967 & 24 & 18 & 42 & 93,3 & 3 & - & - & 3 & 6,7 \\
\hline
\end{tabular}

\section{III. - Types de miels caractéristiques au Luxembourg}

On appelle (c type de miel » une combinaison de pollens caractéristique pour certaines régions et qui permet souvent une localisation serrée de la provenance d'un miel inconnu. Les types de miel résultent de l'influence des conditions géo-botaniques, climatiques, agricoles et forestières d'une région. Pour découvrir ces types, il importe de ne pas se baser uniquement sur les formes de pollens prédominantes, représentées en masse, mais aussi sur les formes d'accompagnement et sur les combinaisons de formes caractéristiques. Les miels luxembourgeois présentent un intérêt particulier par la position du pays entre la France, la Belgique et l'Allemagne, dont les types de miel sont déjà en partie connus.

3. 1. - Combinaisons de pollens caractéristiques dans les miels luxembourgeois et leur répartition.

Comme déjà cité plus haut, le trèfle blanc est au premier plan des sources de nectar dans toutes les régions du Luxembourg et produit souvent des miels unifloraux. Cette constatation rallie le Luxembourg au cercle des pays de l'Europe du Nord, où la récolte sur trèfle blanc est une des plus importantes sources de miel. Les miels de trèfle blanc sont connus en Europe surtout au nord de la France, en Belgique, en Allemagne du Nord, en Hollande, au Danemark, dans les pays baltes, la Scandinavie et la Grande-Bretagne (Maurizio et Louveaux 1965). En Belgique, par exemple, le trèfle blanc est pollen dominant dans $80 \%$ à peu près des échantillons examinés, au Danemark cette proportion dépasse $60 \%$ (Hammer et al. 1948, Martens et al. 1964). Le 
pollen de trèfle blanc se trouve combiné de diverses manières avec d'autres formes de pollen dans les miels luxembourgeois. Les combinaisons avec des crucifères, Rubus, Salix et Myosotis sont particulièrement fréquentes au niveau des pollens dominants et d'accompagnement (voir tableau 5a). On trouve aussi le trèfle blanc allié au pollen d'arbres fruitiers et des deux espèces de Lotus. La combinaison Trifolium repens / Lotus uliginosus est particulièrement caractéristique, parce que cette dernière forme est rare dans les miels d'Europe centrale, tandis qu'elle est fréquente dans les miels de l'Allemagne du Nord, de la Belgique et de la Hollande. Lotus uliginosus qui accompagne généralement Calluna vulgaris dans les miels du nord de l'Europe, est par contre toujours combiné avec le trèfle blanc dans les miels luxembourgeois (Maurizio 1966).

Cette observation souligne un autre signe caractéristique des miels luxembourgeois, le manque presque total de callune dans le spectre pollinique. On ne trouve du pollen de Calluna que dans deux des 130 échantillons, avec $1 \%$ seulement chacun. Le Luxembourg se rallie ainsi aux régions françaises voisines, où Calluna vulgaris ne se trouve que comme pollen isolé, tandis qu'en Allemagne du Nord et en Belgique elle représente une importante récolte tardive et qu'elle se trouve souvent dans le spectre pollinique comme pollen dominant ou d'accompagnement (Evenius 1960, Génier 1966, Martens et al. 1964, Maurizio 1966, Maurizio et Louveaux 1965).

En comparant avec les miels belges, on remarque aussi la rareté du pollen de Castanea sativa dans les miels luxembourgeois. Il se trouve comme pollen isolé seulement dans 7 échantillons, qui tous proviennent des trois villages suivants, Itzig 2, Schengen 4, Mondorf 1. En Belgique, le châtaigner est, après le trèfle blanc, la forme la plus répandue dans le spectre et devient souvent pollen dominant ou d'accompagnement (MARTENs et al. 1964).

Vicia faba et Filipendula ulmaria sont des formes d'accompagnement caractéristiques des miels luxembourgeois qu'on trouve dans plus d'un tiers des échantillons et qui atteignent à l'occasion le rang de pollen dominant ou d'accompagnement. Les miels luxembourgeois ressemblent par là aux belges (et aussi aux danois). Centaurea cyanus, Teucrium, Onobrychis, Chamaenerion, Melilotus et Rhamnus sont caractéristiques mais moins fréquents. La présence de Teucrium scorodonia est frappante, parce que cette forme est relativement rare dans les miels européens, et n'a été trouvée en assez grande quantité jusqu'à maintenant qu'en Allemagne (Westphalie) et en France (Cévennes) (Maurizio et Louveaux 1965, Zander 1937). Malgré la fréquence de Chamaenerion augustifolium au Luxembourg, le pollen de cette plante ne se trouve que dans 14 miels. Cette constatation confirme l'observation générale d'après laquelle le pollen de Chamaenerion n'aboutit que rarement dans le nectar et le miel, ce qui est dû à la conformation et à la biologie de la fleur. Les pollens de Centaurea cyanus, Onobrychis et Melilotus trouvés dans ces miels peuvent être 
considérés comme très caractéristiques. Le pollen de bleuet se trouve dans de nombreux miels européens comme pollen dominant ou d'accompagnement, par exemple en Europe occidentale, orientale et du Nord. Les miels d'esparcette sont surtout répandus dans l'Europe du Sud, du Sud-Est et occidentale. Le Luxembourg se rattache aux régions françaises voisines avec ses pollens d'Onobrychis assez fréquents. Le pollen de Melilotus est en général assez rare dans les miels européens. Des miels purs de mélilot ont été signalés en Allemagne, mais ce sont des cas isolés; dernièrement plusieurs miels de mélilot ont été décrits en Belgique (Louveaux $1970 \mathrm{~b}$, Martens et al. 1964, Maurizio et Louveaux 1965, Zander 1935, 1937). Le pollen de mélilot trouvé dans les miels luxembourgeois, quoique plus rare, indique une certaine ressemblance avec les conditions de récolte belges.

Les pollens de Polygonum bistorta, Impatiens, Lythrum salicaria et Carex ont plutôt une signification locale. Ils correspondent, comme ceux des saules, Filipendula et Rhamnus, à une récolte sur des rives boisées et des marais.

Les tableaux 2 et 5 a donnent aussi des renseignements sur la répartition des formes de pollens dans les miels des différentes régions du Luxembourg. On peut distinguer deux groupes - les formes de pollen qui sont réparties plus ou moins régulièrement dans les échantillons de miel de toutes les régions du pays, et celles qui se trouvent surtout dans les échantillons de certaines régions.

Dans le premier groupe, de répartition générale, on trouve surtout le trè̀le blanc. On peut y ajouter aussi les crucifères, Salix, Trifolium pratense, les deux espèces de Lotus, les arbres fruitiers, Rubus, Filipendula, Myosotis et Taraxacum. Quelques-unes d'entre elles se trouvent concentrées, comme pollens dominants et d'accompagnement, dans certaines zones du pays, par exemple les crucifères dans le sud (cantons $1,4,5$ ) les espèces de Salix dans l'est (cantons 5, 6, 7) et Filipendula dans le centre et l'ouest (cantons 1, 2, 3, 10, 11).

Les formes du second groupe se concentrent, en tant que pollens isolés, dans des régions déterminées du pays, par exemple Melilotus (cantons 4, 6, 11) Chamaenerion (cantons 10, 11, 12) Teucrium (cantons 8, 10, 11) Vicia faba (cantons 1, 4, 5, 10). On peut voir ici aussi la fréquence du miellat qui est en général peu répandu, avec quelques condensations locales (cantons 2, 8, 12, voir tableau 6 a).

Le type de miel dominant au Luxembourg peut généralement être caractérisé par les combinaisons de pollens suivantes : Trifolium repens- crucifèresRubus- arbres fruitiers- Trifolium pratense- Lotus- Filipendula, accompagnés de Teucrium, Onobrychis, Melilotus, Centaurea cyanus, Taraxacum, Rhamnus, Myosotis et une faible proportion d'indicateurs de miellat.

\section{2. - Comparaison des spectres polliniques de miels luxembourgeois d'années différentes.}

La comparaison des échantillons de miels des années 1966 et 1967 avec 
ceux d'années antérieures montre un certain décalage dans le spectre pollinique (tableaux 2, $5 \mathrm{~b}, 6 \mathrm{~b}$ ). La différence réside surtout dans un accroissement notable du trèfle blanc comme pollen dominant (31\% contre $11 \%$ ) et une diminution des crucifères comme pollens dominants et $d$ 'accompagnement ( 1 et $6 \%$ contre 4 et $13 \%$ ). La participation de Trifolium pratense, Onobrychis et Salix est aussi un peu plus basse dans les échantillons récents que dans les anciens. La fréquence de Filipendula et Rubus est, par contre, restée inchangée au long des années. La fréquence du miellat semble être un peu plus grande dans les récoltes 1945 et 1966 (à peu près $21 \%$ des échantillons avec davantage de miellat, contre 12,8 resp. $6,7 \%$ ).

On constate donc un envahissement récent et général par la récolte de trèfle blanc, qui a déjà été constaté dans le nord de la France (Louveaux 1970 a, b). Cette constatation est d'autant plus intéressante que la tendance contraire règne en Scandinavie, c'est-à-dire que la récolte de trèfle blanc, qui prédominait généralement jusqu'à maintenant, est en train d'être supplantée par la récolte des crucifères. Il semble qu'au Luxembourg le changement soit dû à la lutte intensive contre les mauvaises herbes qui fait reculer la moutarde des champs, tandis qu'en Scandinavie, l'accroissement de la récolte des crucifères est en corrélation avec l'introduction de cultures de colza étendues. L'évolution dans les cultures, qui influence celle de la flore mellifère, se reflète ainsi dans le spectre pollinique du miel.

Reçu pour publication en juin 1971.

Eingegangen im Juni 1971.

\section{REMERCIEMENTS}

Cette analyse a été faite à l'instigation de M. J. Poos, Président de la Fédération des Unions d'Apiculteurs du Grand-Duché de Luxembourg, qui a mis à ma disposition un grand nombre d'échantillons du contrôle du miel, et m'a fourni tous les renseignements sur leur provenance ainsi que sur la configuration du pays. J'aimerais le remercier ici vivement pour son aide constante. Je dois aussi des remerciements à Mme Rochat-PÉClaRD qui s'est chargée de la traduction française et à Mme Schneider-Schmitz, qui participa à l'examen microscopique d'une partie des échantillons de miel et prépara les planches 2 et 3.

\section{ZUSAMMENFASSUNG}

Die vorliegende Mitteilung befasst sich mit der mikroskopischen Untersuchung luxemburgischer Honige. Zweck der Untersuchung war, zur Abklärung folgender Fragen beizutragen :

- Von welchen Pflanzen stammen zur Hauptsache die luxemburgischen Honige, d.h. welche Pollenformen herrschen darin vor und von welchen Formen werden sie begleitet?

- Wieweit ist Honigtautracht an luxemburgischen Honigen beteiligt?

- Welche charakteristischen Honigtypen kommen in Luxemburg vor und wie sind sie auf die einzelnen Landesgegenden verteilt? 
- Bestehen Unterschiede im Pollenbild zwischen Honigen aus früheren und neueren Ernten, die auf eine Wandlung der Trachtverhältnisse hinweisen?

Die Untersuchung umfasst 130 Honigproben von Bienenständen in den 12 Kantonen Luxemburgs. Die Proben stammen aus 72 Ortschaften und verteilen sich auf die Ernten der Jahre 1945, 1947, 1954, 1966 und 1967 (Tab. 1, Abb. 1).

Die Honige wurden nach der von der Internationalen Kommission für Bienenbotanik der I.U.B.S. beschriebenen Methodik verarbeitet und die darin gefundenen Pollenkörner und Honigtaubestandteile bestimmt und gezählt. Die Auszählungsresultate wurden nach der Häufigkeit in Gruppen eingeteilt. Für Pollenkörner in drei Gruppen : Leit-, Begleit-und Einzelpollen; für Honigtauelemente in 5 Gruppen : kein, wenig, ziemlich viel, viel und sehr viel.

Die Ergebnisse der Untersuchung sind in den Tabellen 2-6 und den Abbildungen 2 und 3 dargestellt.

\section{Ergebnisse :}

1. Die luxemburgischen Honige sind ziemlich formenreich : über $60 \%$ enthalten 11-15 verschiedene Pollenformen, weitere $31 \%$ 6-10 Formen; der Mittelwert beträgt 12, 7 Pollenformen je Honig. (Tab. 3, Abb. 2).

2. Im ganzen konnten in den luxemburgischen Honigen 67 verschiedene Pollenformen bestimmt werden (Tab. 2 und 4, Abb. 3). Unter den gefundenen Pollenformen dominiert die Gruppe Weissklee (Trifolium repens, Trifolium hybridum), sie fand sich in 127 der 130 Honigproben, d. h. in $97,7 \%$. In mehr als der Hälfte der Proben fanden sich Pollenkörner von : Rotklee (Trifolium pratense), Hornklee (Lotus corniculatus), den Gruppen Himbeere (Rubus idaeus, Rubus fruticosus) und Obstbäume (Pyrus spp., Malus spp., Prunus spp.), Cruciferen (darunter vor allem Ackersenf, Sinapis arvensis), Weiden (Salix spp.) und Vergissmeinnicht (Myosotis spp.).

20-40\% der Proben enthalten Pollenkörner von : Löwenzahn (Taraxacum officinale), Ackerbohne (Vicia faba), Sumpf-Hornklee (Lotus uliginosus), Ahorn (Acer spp.), Kornblume (Centaurea cyanus) und Gamander (Teucrium scorodonia), sowie von Mädesüss (Filipendula ulmaria) und von Windblütlern wie Gräser (Gramineae), Wegerich (Plantago spp.) und Ampfer (Rumex spp.) und einer bisher nicht identifizierten, als “ Unbekannte Form 1 w bezeichneten Form. Alle übrigen Pollenformen blieben auf weniger als $20 \%$ der Proben beschränkt.

3. Unter den gefundenan Pollenformen treten 17 mengenmässig, d. h. auf der Stufe des Leit-oder Begleitpollens, hervor. Eine dominierende Rolle spielt darunter die Gruppe Trifolium repens, die in $42 \%$ der Befunde als Leitpollen und in weiteren $40 \%$ als Begleitpollen auftritt. Weniger häufig fanden sich die übrigen Leguminosen, die Cruciferen, Rosaceen, Salix-Arten und Teucrium scorodonia auf der Stufe des Leit-oder Begleitpollens (Tab. 5a).

4. Honigtaubestandteile fanden sich in zahlreichen luxemburgischen Honigen, in der Regel blieb aber ihr Anteil gering (Tab. 6a). $45 \%$ der Proben enthielten so wenig Pilzsporen, dass sie als reine Blütenhonige angesprochen werden können; in weiteren $41 \%$ der Proben blieb der Honigtauanteil sehr niedrig. Nur 17 Proben (13\%) wiesen grössere Mengen von Honigtauelementen auf, davon 4. Proben « viel » und eine « sehr viel ». Eigentliche Waldhonige waren demnach unter den untersuchten Proben selten.

5. Auf Grund der Untersuchung lassen sich gewisse für Luxemburg charakteristische Pollenkombinationen (Honigtypen) feststellen.

In allen Gegenden Luxemburgs steht Weissklee als Trachtquelle im Vordergrund und liefert oft einseitige Sortenhonige. Damit schliesst sich Luxemburg dem Kreis der nordeuropäischen Länder, mit vorherrschender Weisskleetracht an.

Weisskleepollen tritt in den luxemburgischen Honigen besonders häufig in Kombination mit Cruciferen, Rubus, Salix und Myosotis auf. Häufig ist auch die Kombination von Weissklee 
mit Obstpollen, den beiden Lotus-Arten, Vicia faba und Filipendula ulmaria. Als charakteristisch kann die Verbindung Trifolium repens-Lotus uliginosus gelten, weil letztere Form bisher nur in nordeuropäischen Honigen und hier meist in Kombination mit Calluna vulgaris gefunden wurde. Charakteristisch dürfte auch das seltene Vorkommen des Pollens von Calluna vulgaris und Castanea sativa in luxemburgischen Honigen sein, wodurch sie sich von belgischen Honigen unterscheiden.

Unter den weniger verbreiteten Pollenarten können Centaurea cyanus, Onobrychis viciifolia, Rhamnus spp. und Chamaenerion angustifolium, besonders aber Melilotus spp. und Teucrium scorodonia als typisch für luxemburgische Honige betrachtet werden.

Der allgemeine in Luxemburg verbreitete Honigtypus kann mit folgender Pollenkombination umschrieben werden : Trifolium repens-Cruciferen-Rubus-Obstbäume-Trifolium pratense-Lotus corniculatus-Lotus uliginosus-Filipendula, in Begleitung von Teucrium scorodonia, Melilotus spp., Onobrychis viciifolia, Centaurea cyanus, Taraxacum officinale, Rhamnus spp. und Myosotis spp. und einem schwachen Anteil von Honigtauelementen. Dieser allgemein verbreitete Honigtypus bildet, durch Konzentrationen einzelner Formen, in gewissen Gegenden des Landes charakteristische Nuancen.

6. Die Honige aus den Jahren 1966 und 1967 zeigen, im Vergleich zu denen aus früheren Jahren, gewisse Unterschiede des Pollenspektrums. Der Hauptunterschied beruht auf einer deutlichen Zunahme der Weisskleebefunde auf der Stufe des Leit-und Begleitpollens, bei gleichzeitigem Rückgang der Cruciferenbefunde auf diesen Stufen (Tab. 2, 5b). Diese, auch in Nordfrankreich beobachtete Erscheinung dürfte mit der neuerdings intensivierten Unkrautbekämpfung zusammenhängen, durch welche die Cruciferenbestände (vor allem Ackersenf) zurückgedrängt und die Bienen zu einer Umstellung auf Weisskleetracht veranlasst wurden. Bei den Honigtaubefunden sind im Verlaufe der Jahre keine deutlichen Unterschiede festzustellen (Tab. 6b).

\section{REFÉRENCES BIBLIOGRAPHIQUES}

Evenius J., 1960. Pollenanalytische Prüfung von Honigen aus der Bundesrepublik. D. Bienenwirtschaft, $11 ; 76-80$.

Evenius J., Focke E., 1967. Mikroskopische Untersuchung des Honigs. Handbuch der Lebensmittelchemie, $\mathrm{V}: 560-590$.

Genier G., 1966. Le pollen des Ericacées dans les miels français. Annales de l'Abeille, 9 ; 271 321.

Hammer O., Jorgensen E. G., Mikkelsen V. M., 1948. Studier over danske honingprövers inhold af blomsterstöv, Tidskr. Planteavl. 52; 293-352.

Internationale Kommission für Bienenbotanik der I.U.B.S. 1962, 1963, 1970. Methodik der Honig-Pollenanalyse. Z. Bienenforsch. 6, 115/116, Ann. Abeille 6, 75/76, Bee World 43, 122-124, Apidologie 1, 193-209, 211-227, Bee World 51, 125-238.

Louveaux J., 1956. Étude des miels français par l'analyse pollinique. XVIe Congrès international Apiculture, Vienne.

Louveaux J., 1968. Composition, propriétés et technologie du miel. Traité de Biologie de l'abeille., III, 277-424, Masson Paris.

Louveaux J., 1970a. Mündliche Mitteilung.

Louveaux J., 1970b. Atlas photographique d'analyse pollinique des miels. Annexes microphotographiques aux méthodes officielles d'analyse T. III. Service Répression Fraudes, Paris.

Luxemburg, 1966. Grossherzoglicher Beschluss vom 12. März 1966 über den Honig und gleichartige Produkte. Luxemb. Bienenztg., 81, 85/86.

Martens N., van laere O., Pelerents C., 1964. Studie van de bijenflora in Belgie door pollenanalyse. Biol. Jaarboek, Dodonaea, Gent, 32; 292-324.

Mavrizio A, 1966. Das Pollenbild europäischer Heidehonige. Annales de l'Abeille, 9; 375-387.

Maurizio A., Louveaux J., 1965. Pollens de plantes mellifères d'Europe. UGAF, Paris.

Zander E., 1935, 1937. Beiträge zur Herkunftsbestimmung bei Honig. Pollengestaltung und Herkunftsbestimmung bei Blütenhonig. I. Berlin, II. Leipzig.

Adresse de l'auteur : Dr. A. Maurizio, Rosenweg 9, 3097 Liebefeld, Suisse. 